reference list. All these points might perhaps be taken into account in a second edition.

One of the tasks of the historian of science is to see that credit is properly assigned for scientific discoveries. Professor Brush emphasizes the part played by Wilhelm Lenz, the supervisor of Ernst Ising, in formulating the Ising model; in fact Brush uses the terminology "LenzIsing model". He also tells the sad story of J.J. Waterston who anticipated some of the ideas of Clausius in 1845. Waterston's paper was rejected by the Royal Society and the manuscript was not even returned to the author. In addition, the respective contributions of Bose and Einstein to the development of Bose-Einstein statistics, and of Fermi and Dirac to Fermi-Dirac statistics are described.

A useful feature of the book is the wide and detailed reference list, and research scientists will be surprised to find how the literature on the history of science has grown. For example, I learnt of the existence of English translations of several of Clausius's fundamental papers, and of a publication of Waterston's collected scientific papers. In all, this is a book from which all who are interested in statistical mechanics can benefit.

$\square$

Cyril Domb is Professor of Physics at Bar-Ilan University, Ramat-Gan, Israel.

\section{Managing disease}

\section{J.W. Deacon}

The Nature and Practice of Biological

Control of Plant Pathogens.

By R. James Cook and Kenneth F.

Baker.

American Phytopathological Society:

1983. Pp.539. \$43.

Biol.ogiCAI. control of plant diseases has generated much interest in recent years, in large part because of the efforts of a few workers such as James Cook and Kenneth Baker. The achievements up to 1974 were summarized and discussed in their earlier book, Biological Control of Plant Pathogens; this new work is a companion volume rather than a replacement, special attention being given to developments in the past decade. The book is impressive: quite simply, there has never been a better exposition of the "ecological" approach to plant disease control. The authors draw upon their first-hand experience and wide knowledge, and the result is an authoritative and highly readable account of the subject. It should be welcomed by both specialists and university students in the plant sciences.

The title, however, is surely misleading. In the view of many people, biological control is brought about by a third party (i.e. not by the host or the pathogen), either applied directly or promoted by manipulating other factors. Instead, the authors have defined biological control so broadly that it leads to near-absurdities, for example when we are told (p.391) that the growing of genetically different crop cultivars in neighbouring areas is a form of biological control because it restricts the spread of pathogen races specific to any one cultivar. Further (p.407), the use of lime and inorganic fertilizers are said to be ways to achieve biological control if they affect plant resistance. The term then loses all useful meaning.

Semantics apart, this book is actually about disease management by manipulating the host, pathogen or environment (including microorganisms) to achieve what the authors term "sustainable agriculture". As such, it is timely and thought-provoking, though it could usefully have included chemical control methods, because a sustainable agriculture must harness, rationally, all available resources for plant protection. Let us hope that plant pathologists will follow the entomologists' lead in this respect.

Returning to biological control in a restricted sense, there are several successful techniques that are applied widely in commercial practice. But all too many of the "successes" discussed in this book have never reached the stage of exploitation. and the authors could have been more critical in this respect. For example, their earlier book cited astounding benefits when Bacillus subtilis was applied to seeds of crops in Australia: in field trials, carrot yields were increased by $48 \%$ and oat yields by $40 \%$, even when the untreated crops showed no obvious disease symptoms. Baker was associated with this work, so now we could expect to learn of further developments. Instead, the earlier reports are merely repeated. Why aren't Australian growers using B.subtilis and getting huge yield increases? Was the work abandoned or were subsequent trials inconclusive? In order to justify its title, the book should have addressed such questions. And why cite yet more "successes" in the use of seedapplied microbes to control damping-off diseases? We knew this in the 1950s and there have been regular reports ever since, but the method has not been adopted commercially because we have cheap, dependable and reasonably safe fungicides that give the necessary short-term protection to seedlings.

In short, biological control will achieve more converts if it is seen to be approached with realism and less of an evangelical spirit. But this is a relatively minor point. Cook and Baker's new book will encourage and assist workers in this field, while helping to ensure that the momentum of work on biological control is sustained.

J.W. Deacon is a Lecturer in Microbiology a the University of Edinburgh, currently working at the Citrus and Subtropical Fruit Research Institute, Nelspruit, South Africa.

\section{Clearly intermediate mechanics}

\section{J.W. Humberston}

Theoretical Mechanics.

By E. Neal Moore.

Wiley: 1983. Pp.456.

£28.10, \$37.90.

THERE are already so many books on classical mechanics, that one might well question the need for yet another - the field is not growing in the sense that fundamentally new material is continually being added to it. Theoretical Mechanics deserves attention, however, because the style of presentation and the philosophy behind it are somewhat unusual.

The book is designed for students who are already familiar with the subject matter covered in the usual undergraduate course in basic mechanics and who wish to have an introduction to more advanced topics such as Hamiltonian and Lagrangian mechanics, variational methods and canonical transformations. The author believes that an initial understanding of these topics is more likely to be acquired by practice in solving problems than by studying the formal structure of the subject. Accordingly, he has provided numerous worked examples and sets of problems at the end of each chapter.

The book begins with a brief review of basic Newtonian mechanics, and, in addition to the topics already mentioned, contains chapters on central force motion, rotations, oscillations and stability, and a brief introduction to the special and general theories of relativity. Several of these topics are treated in more elementary books on mechanics, but the presentation here is usually more advanced; for example, the Runge - Lenz vector is introduced in the discussion of the motion of a particle under an inverse square law of force.

Eighty pages are devoted to several mathematical appendices, some of which are rather inappropriate in a book at this level. So while the appendices on tensors and group theory are useful, it hardly seems necessary to give the elementary properties of vector operations, determinants and matrices. Finally, there is a quite comprehensive bibliography and a list of references to articles on mechanics published in the American Journal of Physics.

The book is well produced, contains very few typographical errors and is written in a pleasantly informal style. But, as the author himself admits in the preface, rigour is often sacrificed in the interests of clarity.

J.W. Humberston is a Lecturer in the Department of Physics and Astronomy at University College London. 